\title{
Oscillations Control of Rocking-Block-Type Buildings by the Addition of a Tuned Pendulum
}

\author{
Luca Collini, ${ }^{1}$ Rinaldo Garziera, ${ }^{1}$ Kseniia Riabova, ${ }^{1}$ \\ Mariya Munitsyna, ${ }^{2}$ and Alessandro Tasora ${ }^{1}$ \\ ${ }^{1}$ Department of Industrial Engineering, University of Parma, 43100 Parma, Italy \\ ${ }^{2}$ M. V. Lomonosov Moscow State University, Leninskie Gory 1, Moscow 119991, Russia \\ Correspondence should be addressed to Luca Collini; lucaferdinando.collini@gmail.com
}

Received 31 May 2015; Accepted 20 September 2015

Academic Editor: Chao Tao

Copyright (C) 2016 Luca Collini et al. This is an open access article distributed under the Creative Commons Attribution License, which permits unrestricted use, distribution, and reproduction in any medium, provided the original work is properly cited.

\begin{abstract}
This study deals with the dynamical evolutions exhibited by a simple mechanical model of building, comprising a parallelepiped standing on a horizontal plane. The main goal is the introduction of a pendulum in order to reduce oscillations. The theoretical part of the work consists of a Lagrange formulation and Galerkin approximation method, and dry friction has also been considered. From the analytical/numerical simulations, we derive some important conclusions, providing us with the tools suitable for the design of absorbers in practical cases.
\end{abstract}

\section{Statement of the Problem}

This work deals with the dynamical evolutions played by a simple mechanical model. The model comprises a parallelepiped standing on a horizontal plane. As the plane performs vibrational displacements, the parallelepiped begins to evolve its position and these "parallelepiped movements" constitute the subject of our concern. It is worth noting that this study has to be ascribed to the "applied mathematical sphere of searches." Nevertheless, its motivations firmly belong to the "technical sphere of investigation." Hence, in this introduction, a brief description of the underlying technical problem is presented.

The technical scenarios that provoke these studies originate from earthquakes. Interactions between buildings and earthquakes have been studied for a long time and there is much literature about them. In Figure 1, examples of collapses due to earthquakes are reported. These collapses evidence a rocking-block behavior of the structures, namely, rigid movement of the block with respect to the ground, as illustrated in the scheme of Figure 2. When vibrations of buildings may be assumed (and proved) to be elastic and linear, one may think to reduce them by applying some "appropriate mass" [1]. It is in fact possible to absorb energy at certain frequencies (or ranges) by coupling an oscillator with the main structure. This oscillator is often coupled by a spring-damper system, in order to sink energy from the system. However, when considering masonry buildings or no-tension structures in general, the situation changes considerably. Consider, for example, a slender tower, such as the masonry chimney illustrated in Figure 3. When the tower oscillates, for example, under the forcing of an earthquake, a plausible model for its motion is that of a rigid parallelepiped tilting back and forth on a horizontal plane. In this situation, oscillations are no longer linear.

Following the seminal work by Housner in 1963 [2], the problem of the motion of an oscillating parallelepipedshaped rigid body, the so-called "rocking-block" model, has been widely investigated. The main issue investigated in this and other earlier studies [3-11] was the performance and the overturning of a rigid body against the base motion dynamics, for example, an earthquake, assuming the block to be a building model. The work by Housner was devoted to the explanation of the collapse of many bulky structures during the catastrophic Chilean earthquake of May 1960. From the solution of the free oscillation problem of a rigid rocking block bouncing on its vertices, it was demonstrated that tall slender structures show a better stability than expected. 


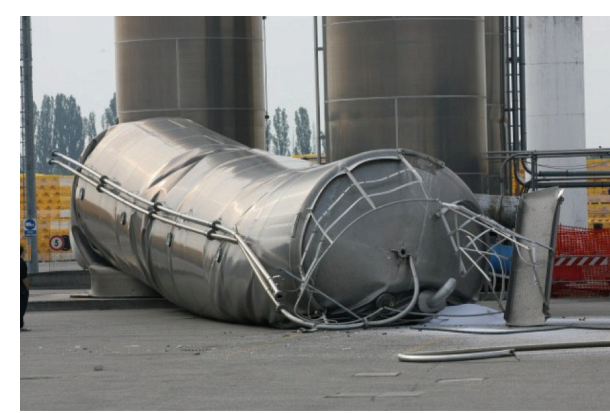

(a)

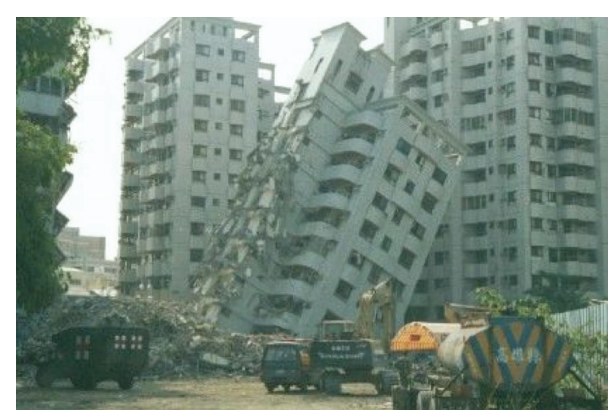

(b)

FIGURE 1: Catastrophic collapses due to earthquakes.

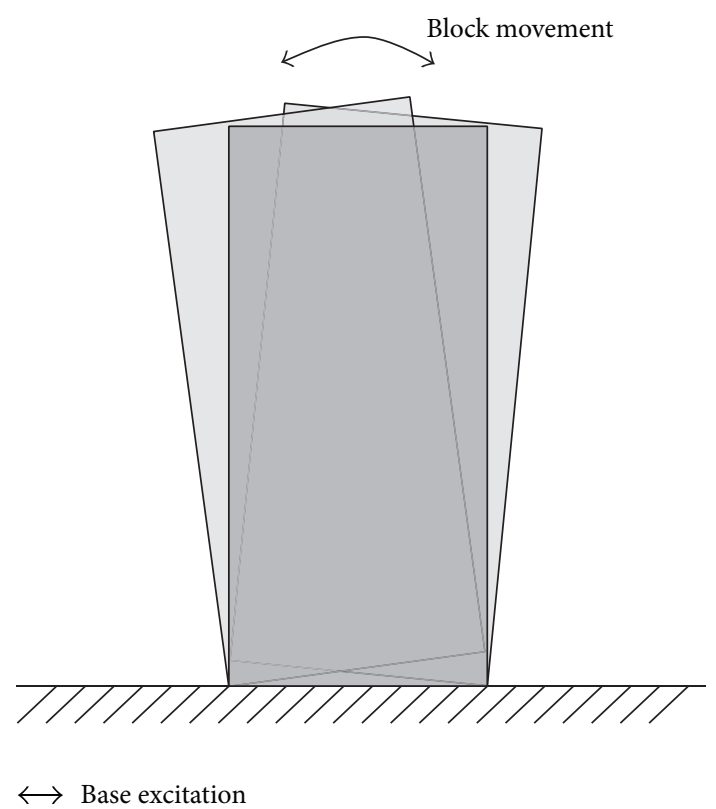

FIgURE 2: Scheme of a rocking block under excitation of the base.

In subsequent years, the works by Hogan [12-15] refined the pioneering study by Housner, assuming a similar rockingblock model and analyzing in depth its dynamics under harmonic forcing. Here, the simple block model is shown to possess extremely complicated dynamics, including chaos. The existence and the form of subharmonic and asymmetric responses are explained and validated by experimental works by Wong and Tso [16]. A rocking-block model has also been employed to study the response of structures, such as columns or monumentary walls subjected to frictionless multiple impacts [17], showing that one may have single and/or simultaneous multiple collisions at the surface contact points. Furthermore, recently, the dynamic response of the rocking block subjected to base excitation has been revisited to offer new closed-form solutions and original similarity laws that shed light on the fundamental aspects of the original model [18].

Finally, similar to the concern of the present work, the problem of vibration control is investigated in $[19,20]$.

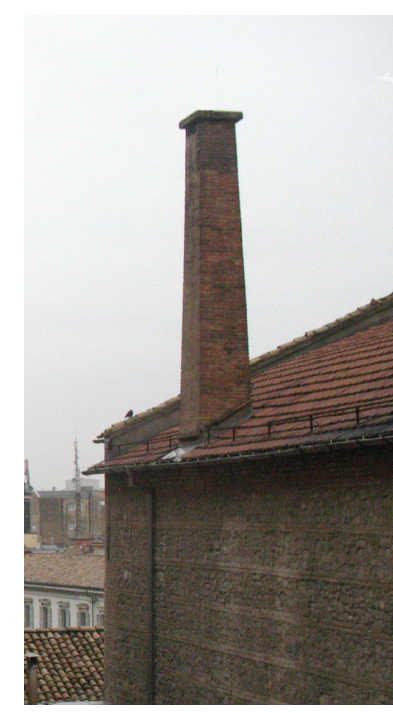

FIGURE 3: An old masonry chimney.

In these works, a systematic theoretical investigation of control/anticontrol of the nonlinear dynamics of a rocking block has been made through the analysis of two curves: the heteroclinic bifurcation and the immediate overturning thresholds, characterizing the system response in excitation parameters space in terms of overturning behavior.

Now, in line with previous investigations, the preliminary questions addressed by the present work are as follows. (i) Is it possible to give some parameterization of these oscillations? (ii) Is it possible to tune a pendulum with this tower so that oscillations are reduced and controlled? (iii) Is it possible to give some sort of frequency response (with all the limitations imposed by nonlinearity)? The simple model studied in the following sections partially answers these questions. The model is somewhat simplified. Plane movement is assumed and, therefore, we name it a parallelepiped and the structure is actually schematized as a "physical rectangle." From this simple model and its elaboration through adding a planar physical pendulum and some damping between the parallelepiped and the ground surface, some interesting results have been achieved. 


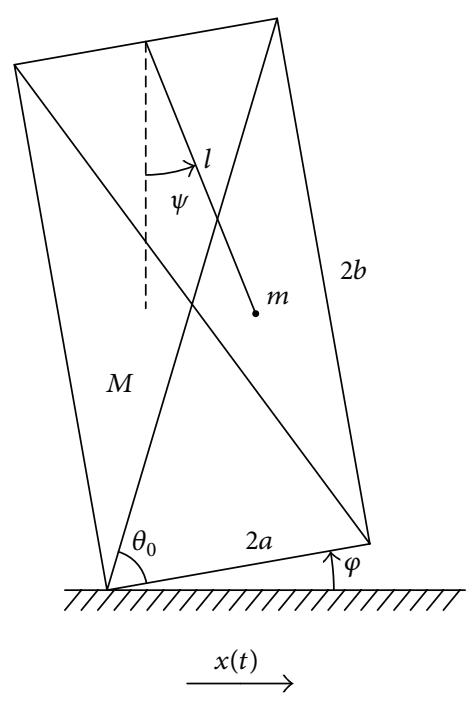

(a) $\varphi>0$

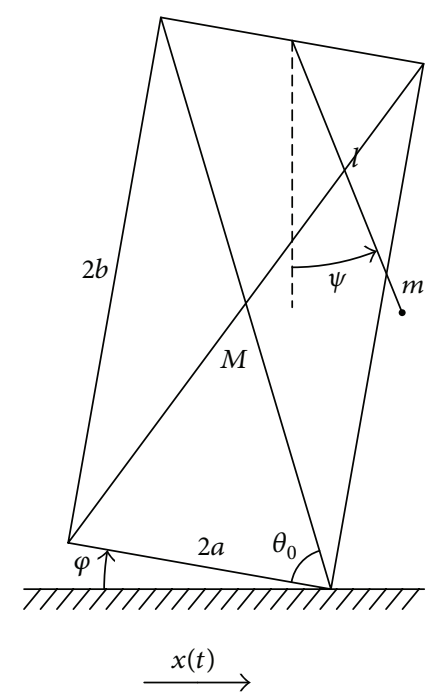

(b) $\varphi<0$

FIGURE 4: The rocking block with the added pendulum, scheme of notations.

\section{Equations of Motion}

The model of block with the pendulum we take into account for the study is shown in Figure 4. With the nomenclature reported in Figure 4, we can write the following geometrical relations of a block of mass $M$ rocking on its corners, to which a pendulum of mass $m$ is added:

$$
\begin{aligned}
r_{0}^{2} & =a^{2}+b^{2}, \\
r_{1}^{2} & =a^{2}+4 b^{2}, \\
\sin \theta_{0} & =\frac{b}{r_{0}}, \\
\cos \theta_{0} & =\frac{a}{r_{0}}, \\
\sin \theta_{1} & =\frac{2 b}{r_{1}}, \\
\cos \theta_{1} & =\frac{a}{r_{1}} .
\end{aligned}
$$

Note that we have two different situations depending on the sign of $\varphi$ angle $(\varphi>0, \varphi<0)$. We assume that the surface on which the block is oscillating moves in accordance with a sinusoidal law:

$$
x=\alpha \sin (\omega t),
$$

hence forcing the block oscillations with amplitude $\alpha$ and frequency $\omega$. The kinetic and potential energies of the block and of the pendulum can be written as

$$
\begin{aligned}
T_{M} & =\frac{M}{2}\left\{\left[\dot{x}-r_{0} \dot{\varphi} \sin \left( \pm \varphi+\theta_{0}\right)\right]^{2}\right. \\
& \left.+\left[r_{0} \dot{\varphi} \cos \left( \pm \varphi+\theta_{0}\right)\right]^{2}\right\}+\frac{J}{2} \dot{\varphi}^{2},
\end{aligned}
$$

$$
\begin{aligned}
T_{m} & =\frac{m}{2}\left\{\left[\dot{x}-r_{1} \dot{\varphi} \sin \left( \pm \varphi+\theta_{1}\right)+l \dot{\psi} \cos \psi\right]^{2}\right. \\
& \left.+\left[r_{1} \dot{\varphi} \cos \left( \pm \varphi+\theta_{1}\right)+l \dot{\psi} \sin \psi\right]^{2}\right\}, \\
V_{M} & =M g[a \sin ( \pm \varphi)+b \cos \varphi], \\
V_{m} & =m g[a \sin ( \pm \varphi)+2 b \cos \varphi-l \cos \psi],
\end{aligned}
$$

in which $J$ is the moment of inertia around the axis of rotation. The signs \pm are valid for both conditions of oscillation. After Lagrangian derivation, we obtain the equations of motion:

$$
\begin{aligned}
& \left(M r_{0}^{2}+m r_{1}^{2}+J\right) \ddot{\varphi} \\
& \quad-m l[ \pm a \sin (\varphi-\psi)+2 b \cos (\varphi-\psi)] \ddot{\psi} \\
& +m l[ \pm a \cos (\varphi-\psi)-2 b \sin (\varphi-\psi)] \\
& \cdot \dot{\psi}^{2}+\alpha \omega^{2}[ \pm(M+m) a \sin \varphi+(M+2 m) b \cos \varphi] \\
& \cdot \sin (\omega t) \\
& +g[ \pm(M+m) a \cos \varphi-(M+2 m) b \sin \varphi]=0 \\
& -[ \pm a \sin (\varphi-\psi)+2 b \cos (\varphi-\psi)] \ddot{\varphi}+l \ddot{\psi} \\
& -[ \pm a \cos (\varphi-\psi)-2 b \sin (\varphi-\psi)] \dot{\varphi}^{2}-\alpha \omega^{2} \\
& \cdot \sin (\omega t) \cos \psi+g \sin \psi=0 .
\end{aligned}
$$

Equations (4) can be solved numerically to obtain angles $\varphi$ and $\psi$ defining the oscillations of the block and of the pendulum in time. 


\section{Oscillations without/with Damping}

3.1. No Damping Problem. In order to study our blockpendulum system, it is interesting at first to consider the motion of the parallelepiped after the end of short excitation given by the base movement (nonforced linearized undamped case) and during excitation of the base and steady motion (forced linearized damped case). Viscous damping is introduced in order to take into account generic damping effects, for example, impact dissipation, internal damping of the material, and air drag. We underline that impact dissipation, which is sometimes considered via the introduction of a coefficient of restitution, has been here considered embedded into viscous damping. A sort of correspondence between the two, that is, damping and coefficient of restitution, can be heuristically carried out from real observations of the oscillation decay.

The calculation of this section gives us results that are in accordance with [2] and validate our nondimensional model. We underline that we obtained these results following an approach that is quite different from [2].

When, after short forcing excitation at the base, the following conditions are satisfied ( $T$ is the period of oscillation):

$$
\begin{aligned}
\alpha \omega^{2} & >\frac{g a}{b} \\
T & >\frac{1}{\omega} \arcsin \frac{g a}{b \alpha \omega^{2}},
\end{aligned}
$$

the parallelepiped will have nonzero velocity and nonharmonic oscillations will start. The equation of motion takes the form

$$
\ddot{\varphi}-\omega_{0}^{2} \sin \varphi=\mp \omega_{0}^{2} \nu \cos \varphi,
$$

where $\omega_{0}=\sqrt{b g /\left(a^{2}+b^{2}+c^{2}\right)}$ is the "natural" frequency of a parallelepiped pinned at the center of the base, $c$ is the radius of inertia of the parallelepiped $(J / M)$, and $v=a / b$ indicates the dimensionless "width" of the parallelepiped.

From a simple geometrical consideration, the boundary of nonoverturning of the block is defined by the expression $\varphi \leq \arctan (\nu)$. Our study considers a slender parallelepiped $(a \ll b)$ and very small angles of motion $(\varphi \ll 1)$.

If it is rewritten in dimensionless time $\tau=t \omega_{0}$, then linearized equation (6) takes the form

$$
\ddot{\varphi}-\varphi=\mp \nu .
$$

Let us now introduce the quantity $\Phi$ indicating the amplitude of the natural oscillations of the block and $\Omega_{0}$ its frequency. Also, consider that, at time $t=0$, the following conditions are satisfied:

$$
\begin{aligned}
& \varphi(0)=\Phi>0 \\
& \dot{\varphi}(0)=0 .
\end{aligned}
$$

Then, at time interval $t \in\left[0, t_{1}\right]$, where $t_{1}=\pi /\left(2 \Omega_{0}\right)$ is a quarter of the oscillation period, the motion can be described by the equation

$$
\ddot{\varphi}-\varphi=-\nu
$$

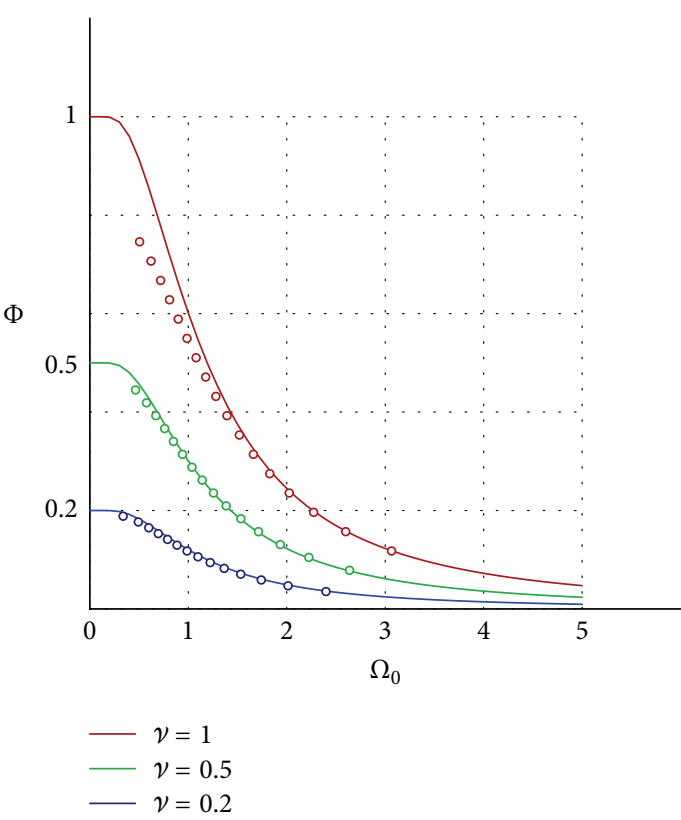

FIGURE 5: Amplitude of oscillation versus frequency of oscillation at various $\nu=a / b$ ratios.

The solution of (9), with the initial conditions (8), becomes

$$
\varphi(t)=v+(\Phi-v) \cosh (t)
$$

which also satisfies the condition $\varphi\left(t_{1}\right)=0$, from which the following dependence of the amplitude of oscillation from its frequency can be derived:

$$
\Phi=\nu\left(1-\cosh ^{-1} \frac{\pi}{2 \Omega_{0}}\right)
$$

This function is plotted in Figure 5 for different values of the parameter $v$ (solid lines). Points in the plot of Figure 5 report the results of the numerical integration of the equations of motion (4). For large values of $v$, that is, for wide blocks, the plot of (11) and the results of the numerical integration are in agreement only in the field of small amplitudes.

These results are somewhat in accordance with those reported in previous works $[2,14]$.

Let us notice that, from (10), it is possible to find the angular velocity $\dot{\varphi}_{1}=\dot{\varphi}\left(t_{1}\right)$ of the block in correspondence with the sign change in the equation of oscillations (7) and to proceed with the second quarter of the period of oscillation, integrating the equation $\ddot{\varphi}-\varphi=+\nu$ with the new entry condition $\varphi\left(t_{1}\right)=0, \dot{\varphi}\left(t_{1}\right)=\dot{\varphi}_{1}$.

3.2. Steady-State Problem. Now, let us consider the problem of the steady motion of the parallelepiped on a vibrating surface. Such a regime of motion originates in the real world during earthquakes and, therefore, we introduce dissipation into the model, as described in the previous section, in order 
to make the model more realistic. For example, dissipation can be considered in the following equation of motion:

$$
\begin{gathered}
\left(a^{2}+b^{2}+c^{2}\right) \ddot{\varphi}+\alpha \omega^{2}[ \pm a \sin \varphi+b \cos \varphi] \sin (\omega t) \\
+g( \pm a \cos \varphi-b \sin \varphi)=0
\end{gathered}
$$

by adding the term $k \operatorname{sgn}(\dot{\varphi})$, with $k=\kappa / \omega_{0}^{2}$, where $\kappa$ is the constant of "dry friction." In dimensionless time, the equation of motion of the parallelepiped results as

$$
\begin{aligned}
\ddot{\varphi}+ & k \operatorname{sgn}(\dot{\varphi})-\sin \varphi-f \nu \sin \varphi \cos \xi t \\
& =\mp \nu \cos \varphi+f \cos \varphi \cos \xi t,
\end{aligned}
$$

where $f=\alpha \omega^{2} / g$ is the dimensionless amplitude of the exciting force and $\xi=\omega / \omega_{0}$ is the dimensionless frequency of oscillation.

If parameter $\nu$ is small enough, and angle $\varphi$ is consequently small as well, the last term in the left part of (13), namely, $\nu \sin (\varphi)$, is of the second order of infinitesimal and, hence, negligible. The linearized equation (13) becomes

$$
\ddot{\varphi}+k \operatorname{sgn}(\dot{\varphi})-\varphi=\mp \nu+f \cos \xi t .
$$

As the aim of the present work is to investigate the worst cases of forcing conditions, such as the source of resonance provided by earthquakes, let us assume that the frequency of the block oscillations $\Omega$ coincides with the frequency of the exciting force; that is, $\Omega=\xi$. Let the initial conditions at $t=0$ be

$$
\begin{aligned}
& \varphi(0)=\Phi>0 \\
& \dot{\varphi}(0)=0 ;
\end{aligned}
$$

then, in the time interval $t \in\left[0, t_{1}\right]$, where $t_{1}=\pi /\left(2 \Omega_{0}\right)$, the equation of motion becomes

$$
\ddot{\varphi}-\varphi=-\nu+k+f \cos (\xi t-\gamma)
$$

and in the time interval $t \in\left[t_{1}, 2 t_{1}\right]$

$$
\ddot{\varphi}-\varphi=\nu+k+f \cos (\xi t-\gamma) \text {. }
$$

Here, $\gamma$ denotes the phase difference between the oscillation of the parallelepiped and the motion of the base.

The equation of motion (16) with the initial conditions expressed by (15) can be written as

$$
\begin{aligned}
\varphi(t)= & v-k+\left(\Phi-v+k+\frac{f \cos \gamma}{1+\xi^{2}}\right) \cosh t \\
& +\frac{\xi f \sin \gamma}{1+\xi^{2}} \sinh t-\frac{f}{1+\mu^{2}} \cos (\xi t-\gamma),
\end{aligned}
$$

where the dimensionless parameter $\mu=m / M$ is introduced. From this law of motion, it is possible to find out the angular velocity $\dot{\varphi}_{1}=\dot{\varphi}\left(t_{1}\right)$ at which the block changes in sign. Integrating (17) with the initial conditions $\varphi\left(t_{1}\right)=0$ and $\dot{\varphi}\left(t_{1}\right)=\dot{\varphi}_{1}$, we will determine the motion of the parallelepiped during the second interval $t \in\left[t_{1}, 2 t_{1}\right]$, depending on the amplitude $\Phi$ and on the phase $\gamma$ of steadystate oscillations.

Additionally, because in the steady-state motion the equalities $\varphi\left(2 t_{1}\right)=-\Phi$ and $\dot{\varphi}\left(2 t_{1}\right)=0$ hold, the amplitude and the phase of oscillations can be determined, depending on the excitation parameters, as follows:

$$
\begin{aligned}
\sin \gamma & =\frac{k\left(1+\xi^{2}\right) \sinh (\pi / 2 \Omega)}{f \cosh (\pi / 2 \Omega)} \\
\Phi & =\nu-k-\frac{f \cos \gamma}{1+\xi^{2}} \\
& +\frac{-\nu+k+\left(f \sin \gamma /\left(1+\xi^{2}\right)\right)(1-\xi \sinh (\pi / 2 \Omega))}{\cosh (\pi / 2 \Omega)} .
\end{aligned}
$$

Note that (19) tends to (11) when $k=\xi=f=0$. From (19), using as nondimensional parameters $v=0.2, k=0.05$, and $f=0.1$, we can obtain the curves displayed in Figures 6(a) and 6(b). In $\Phi-\xi$ graph of Figure 6(a), the bold solid curves in blue represent the amplitude-frequency and the phase-frequency characteristics corresponding to the resonance condition $\Omega=\xi$; the same curves extrapolated for very small $\xi$ are drawn in grey color. In the same plots, the thin curves represent the same characteristics corresponding to the frequency of excitation $\Omega=\xi / 3$, obtained for a bigger initial tilt angle. The dashed line is the free vibration curve, equation of motion (11), included as a reference. In the same figure, points designate the results obtained via the numerical integration of the equations of motion, validating (19). Also, illustrated in Figure 6(a) is $\gamma-\xi$ graph, which represents the phase characteristics. Figure 6(b) encloses the behavior just described above, for initial conditions leading to $\Omega=\xi / 2$ and $\Omega=\xi / 4$.

It is clear that formula (19) greatly supports the design of a block, by giving a clear insight of the amplitude of motion when in the presence of oscillations of the base. The identification of the most dangerous components in earthquakes might not be a straightforward task; however, the range of frequencies never goes out of the range of validity of this formula.

3.3. Resolution by Bubnov-Galerkin's Method. Now, we present a short development of the previously treated problem via Bubnov-Galerkin's method. The quantities $\nu$ and $\varphi$ no longer need to be very small. In order to simplify the calculations, we will use an unknown phase of oscillation $\gamma$ and we will write (13) in the form $F(\varphi)=0$, where

$$
\begin{aligned}
F(\varphi)= & \varphi^{\prime \prime}+k \operatorname{sgn}\left(\varphi^{\prime}\right)-\varphi \pm f \nu \varphi \cos (\xi \tau+\gamma) \pm \nu \\
& -f \cos (\xi \tau+\gamma) .
\end{aligned}
$$

Using one term of the Fourier series, we will determine the motion in the form

$$
\varphi=\Phi \cos (\xi \tau)
$$



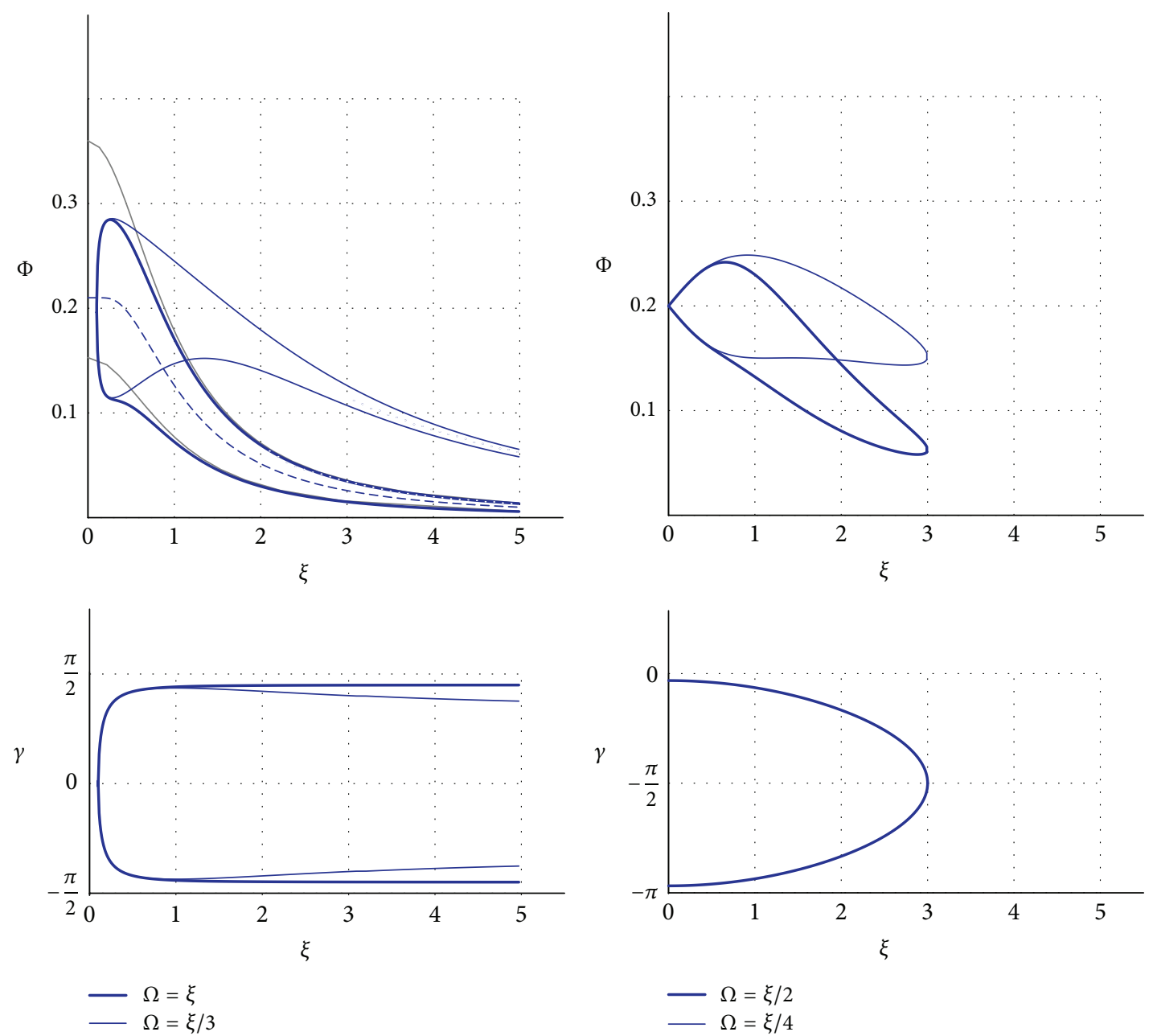

(a)

(b)

Figure 6: Amplitude- and phase-frequency characteristics of the system at different ratios $\Omega / \xi$.

Applying the procedure of orthogonalization of basic functions, that is, from the conditions

$$
\begin{aligned}
& \int_{0}^{2 \pi / \omega} F(\varphi) \sin (\omega \tau) d \tau=0, \\
& \int_{0}^{2 \pi / \omega} F(\varphi) \cos (\omega \tau) d \tau=0,
\end{aligned}
$$

and taking into account (21), it is possible to obtain the equations of the phase $\gamma$ of the system in the form

$$
\begin{aligned}
& \cos \gamma=\frac{4 \nu-\pi \Phi\left(1+\xi^{2}\right)}{f(\pi+(8 / 3) \nu \Phi)}, \\
& \sin \gamma=\frac{4 k}{f(\pi+(4 / 3) \nu \Phi)} .
\end{aligned}
$$

Here, conditions (22) have been used with the following equalities:

$$
\begin{aligned}
& \int_{0}^{2 \pi / \omega} \operatorname{sgn}[\sin (\omega \tau)] \cos (\omega \tau) d \tau=0, \\
& \int_{0}^{2 \pi / \omega} \operatorname{sgn}[\cos (\omega \tau)] \sin (\omega \tau) d \tau=0,
\end{aligned}
$$

$$
\begin{aligned}
\int_{0}^{2 \pi / \omega} \operatorname{sgn}[\sin (\omega \tau)] \sin (\omega \tau) d \tau & =-4, \\
\int_{0}^{2 \pi / \omega} \operatorname{sgn}[\cos (\omega \tau)] \cos (\omega \tau) d \tau & =4, \\
\int_{0}^{2 \pi / \omega} \operatorname{sgn}[\cos (\omega \tau)] \cos ^{3}(\omega \tau) d \tau & =\frac{8}{3}, \\
\int_{0}^{2 \pi / \omega} \operatorname{sgn}[\cos (\omega \tau)] \sin ^{3}(\omega \tau) d \tau & =0, \\
\int_{0}^{2 \pi / \omega}[\cos (\omega \tau)] \sin (\omega \tau) \cos ^{2}(\omega \tau) d \tau & =0, \\
\int_{0}^{2 \pi / \omega} \operatorname{sgn}[\cos (\omega \tau)] \sin ^{2}(\omega \tau) d \tau & =\frac{4}{3} .
\end{aligned}
$$

The characteristics of amplitude-frequency and of phasefrequency are shown in Figures 7 (a) and 7(b), respectively, for the parameters $v=0.2$ and $f=0.1$ and various friction coefficients $k$. From Figure 7 , it can be seen that, 


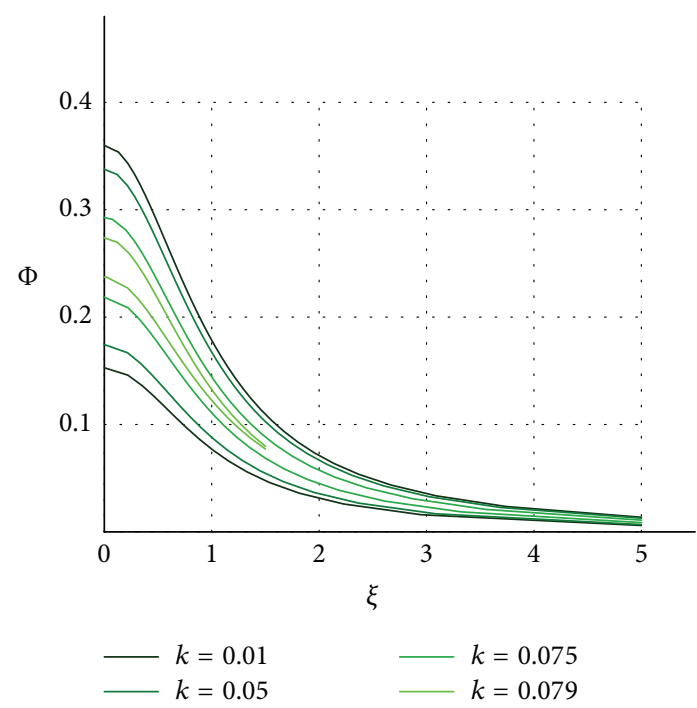

(a)

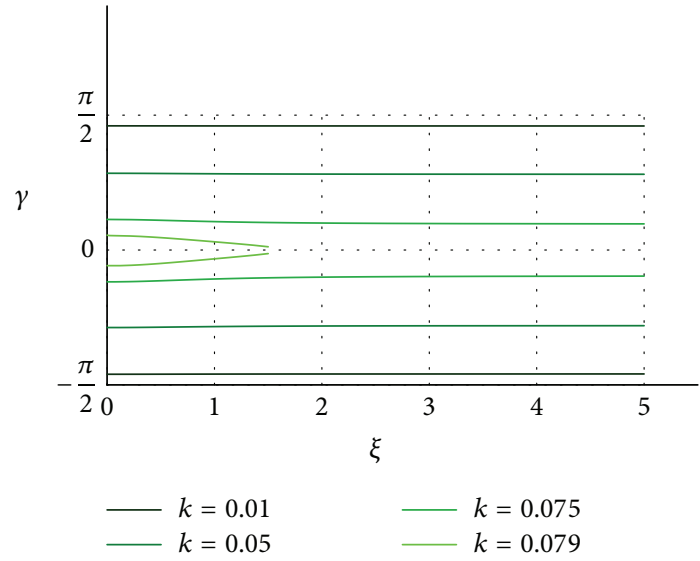

(b)

Figure 7: (a) Amplitude-frequency and (b) phase-frequency characteristics of the system at various friction coefficients $k$, for $v=0.2$ and $f=0.1$.

with the increasing of the dry friction coefficient $k$, a branch of the amplitude-frequency characteristic appears, reflecting in the phase-frequency characteristic around the value $\gamma=$ $\pi / 2$. On the other hand, when the friction coefficient is high enough, the oscillations are absent. Figure 7 synthetizes, with a different degree of approximation, the results already plotted in Figure 6.

\section{Rocking Block with the Pendulum}

The main object of this study is to investigate some device or system suitable for reducing oscillations during earthquakes. Hence, using most of the results calculated in the previous sections, we now consider a system, which includes a pendulum of mass $m$. In the following approach, we will use Bubnov-Galerkin's method and, at this stage, we will not consider friction.

The linearized equations of oscillation of such a system in dimensionless time are

$$
\begin{aligned}
& {\left[1+\mu \rho\left(4+\nu^{2}\right)\right] \varphi^{\prime \prime}-2 \mu \rho \lambda \psi^{\prime \prime}-(1+2 \mu) \varphi} \\
& \quad \pm(1+\mu) \nu \\
& \quad+f[ \pm(1+\mu) \nu \rho+(1+2 \mu)] \sin (\xi \tau)=0 \\
& -2 \rho \ddot{\varphi}+\lambda \rho \ddot{\psi}+f \sin (\xi \tau)+\psi=0,
\end{aligned}
$$

where the following dimensionless parameters are introduced: $\lambda=l / b$ and $\rho=b^{2} / J$.

Let us apply Bubnov-Galerkin's method and we will obtain the solution in the form

$$
\begin{aligned}
& \varphi=\Phi \cos (\xi \tau), \\
& \psi=\Psi \cos (\xi \tau) .
\end{aligned}
$$

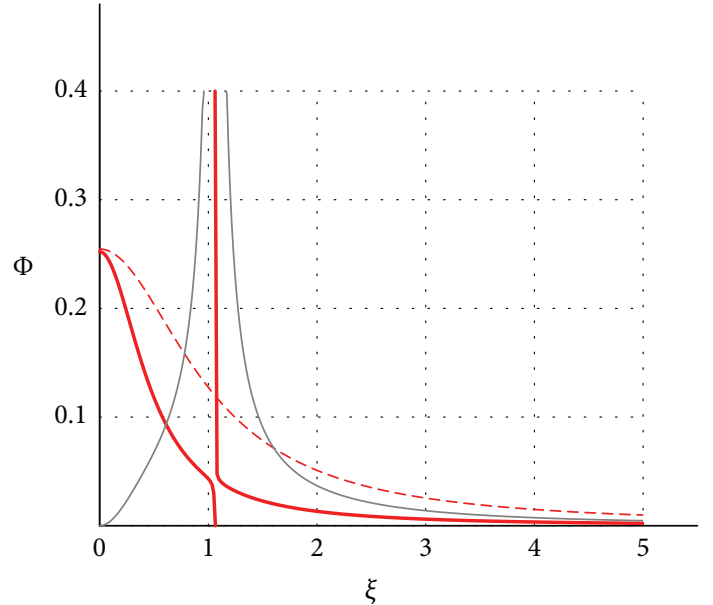

FIGURE 8: Amplitude of free oscillation of the block (bold red line), of the pendulum (thin grey line), and of the block without the pendulum (dashed line) for $v=0.2, \mu=0.01$, and $\lambda=0.1$.

The set of equations describing the oscillation amplitude $\Phi$ of the block and the oscillation amplitude $\Psi$ of the pendulum are, respectively,

$$
\begin{aligned}
& {\left[1+2 \mu+(1+\mu \rho) \xi^{2}+\frac{8}{3} \nu f(1+\mu)\right] \Phi+2 \lambda \rho \mu \xi^{2} \Psi} \\
& \quad=f(1+\mu)-\frac{4}{\pi} \nu(1+\mu) \\
& -2 \rho \xi^{2} \Phi+\left(1-\lambda \rho \xi^{2}\right) \Psi=-f .
\end{aligned}
$$

In the case of $f=0$, (27) describes the dependence of the natural oscillation amplitude of the rocking block with pendulum versus frequency. This dependence is plotted in Figure 8 for the parameters $\nu=0.2, \mu=0.01$, and $\lambda=1$. 


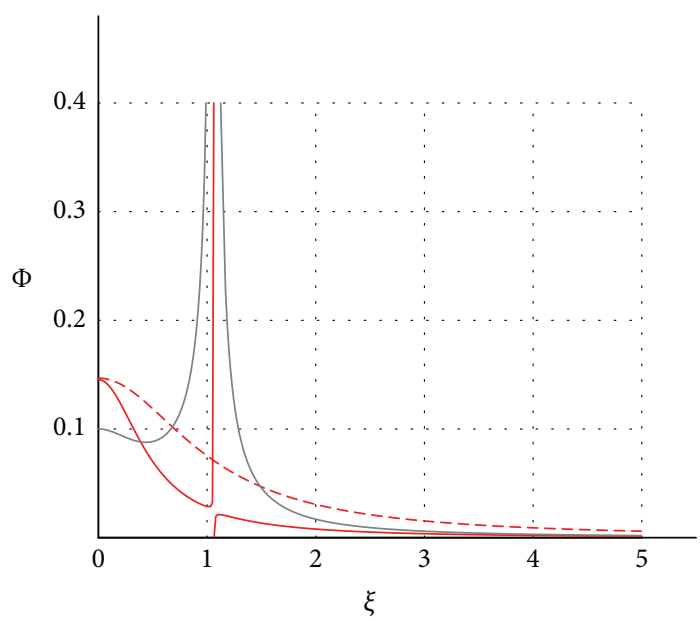

(a)

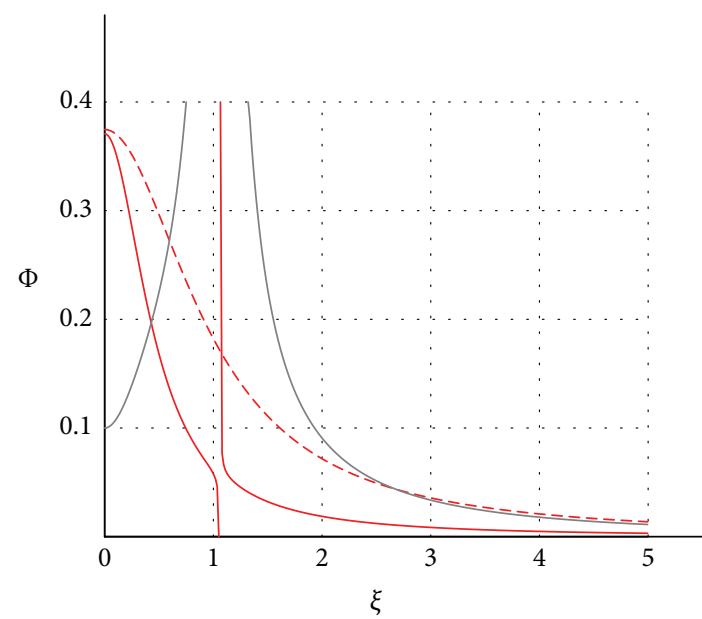

(b)

FIGURE 9: Amplitude of forced oscillation of the block for different slenderness: (a) $\nu=0.15, \mu=0.01, \lambda=0.1$, and $f=0.1$; (b) $\nu=0.40$, $\mu=0.01, \lambda=0.1$, and $f=0.1$.

The bold solid curve in Figure 8 represents the amplitude of the oscillation of the block, while the solid thin curve is the amplitude of the pendulum oscillations. The dotted curve shows the dependence of the block amplitude from frequency in the case of no pendulum, as already shown in Figure 6. Hence, it is shown that the presence of the pendulum can drastically reduce the amplitude of the oscillation of the parallelepiped when its frequency of oscillations does not lie within the pendulum resonance area.

Similar curves can be plotted for the case of forced oscillations; for example, for $f=0.1$, the system behaves as depicted in Figures 9(a) and 9(b). Here, the dotted curves represent the two branches of the amplitude-frequency characteristics of the parallelepiped without the addition of a pendulum (see Figure 7(a)). From these plots, it is deduced that the presence of the pendulum can reduce the amplitude of the oscillation of the block, even under forced oscillations at the base, when the pendulum's natural frequency of oscillation is marginally excited.

In order to better support the theory contained in this work, we have carried out a realistic simulation via a research level multibody dynamic simulator specifically developed by one of the authors, and this simulation has been addressed to a real rocking-block case that is reported in the next section.

\section{A Case Study}

In this section, we present numerical simulations of rocking blocks equipped with pendulums and we compare the outcome of the simulations with the prediction of the analytical approach discussed in this paper.

From a numerical point of view, a model of a rocking block connected with a pendulum leads to a multibody problem involving both bilateral constraints (the hinge between the pendulum and the block) and unilateral contacts that might experience impacts and stick-slip phenomena and, thus, requires numerical schemes for nonsmooth dynamic problems. A conventional strategy for the solution of such a class of problems is based on the regularization of discontinuous terms, which are approximated by Lipschitzcontinuous mollifiers. This casts the original problem into conventional Ordinary Differential Equations (ODEs) or Differential Algebraic Equations (DAEs) that can be solved by well-known numerical integrators. However, a drawback of such regularization approaches is that regularization could lead to extremely stiff functions that hinder the efficiency of ODE/DAE solvers; consequently, very short time steps or sophisticated implicit integrators are required. Therefore, as an alternative to regularization, we use a more advanced mathematical framework that deals directly with the discontinuous nature of friction and contacts, expressing the multibody problem with the tools of Differential Variational Inequalities (DVIs) [21]. In our Chrono::Engine multibody simulation software, we endorse the DVI formulation and, thus, obtain high computational efficiency, good robustness, and numerical stability when simulating problems of multibody frictional contacts. The DVI problem is solved by means of a time-stepping scheme, which requires the solution of a convex second-order Cone Complementarity Problem (CCP) at each time step. In general, CCP problems include the more popular Linear Complementarity Problems (LCPs) as subcases; as for LCPs, there are theoretical results for the existence and uniqueness of the solution under mild assumptions [22]. We solve the CCPs using either a fixedpoint iteration [23] or a Spectral Projected Gradient (SPG) Barzilai-Borwein method.

Impacts between rigid shapes can be handled via the introduction of restitution coefficients, but, if preferred, our software can also handle the case of nonrigid frictional contacts, which fit in the broad context of DVIs. More details on this can be found, for instance, in [24].

The three-dimensional simulation of the rocking block has been performed by introducing three rigid bodies, namely, the moving floor, the block (with dimensions $0.1 \mathrm{~m}$, $0.2 \mathrm{~m}$, and $0.4 \mathrm{~m}$ ), and the pendulum, this being connected by a spherical joint placed at the top of the block. In addition, two box collision shapes have been assigned to the block 

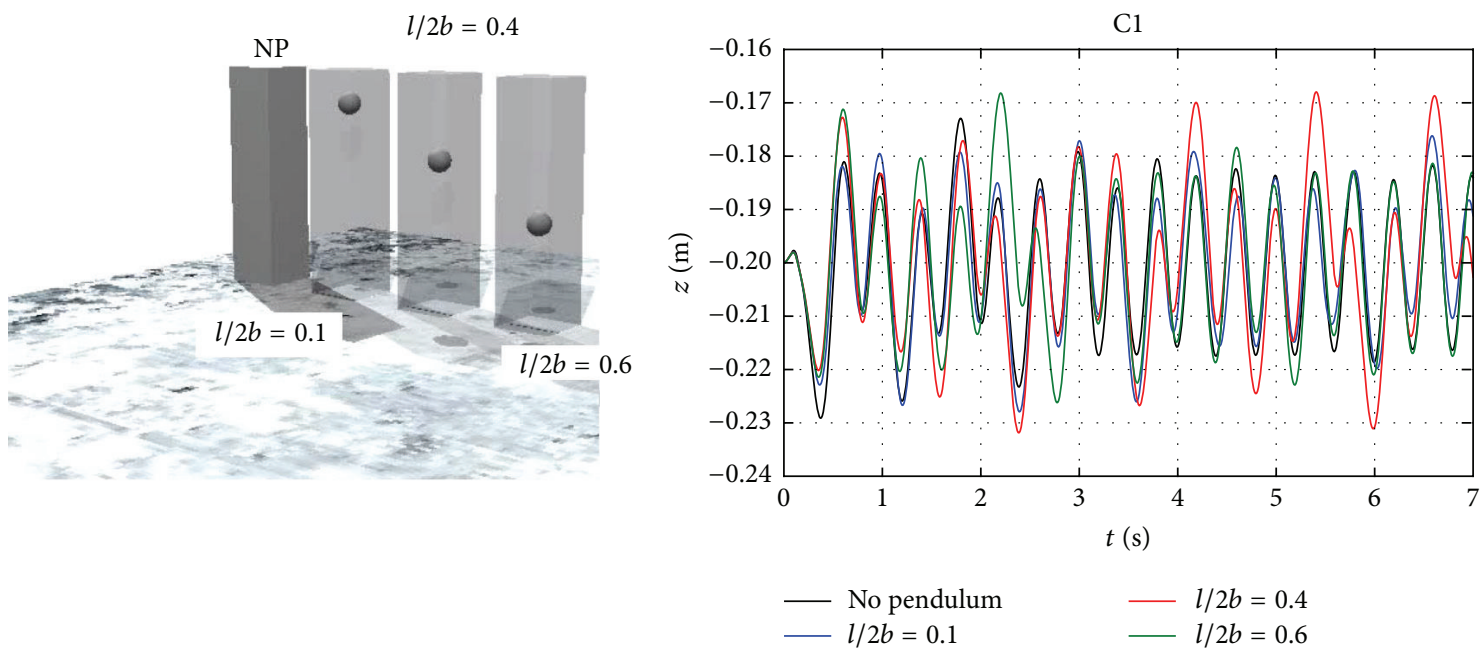

(a)

(b)
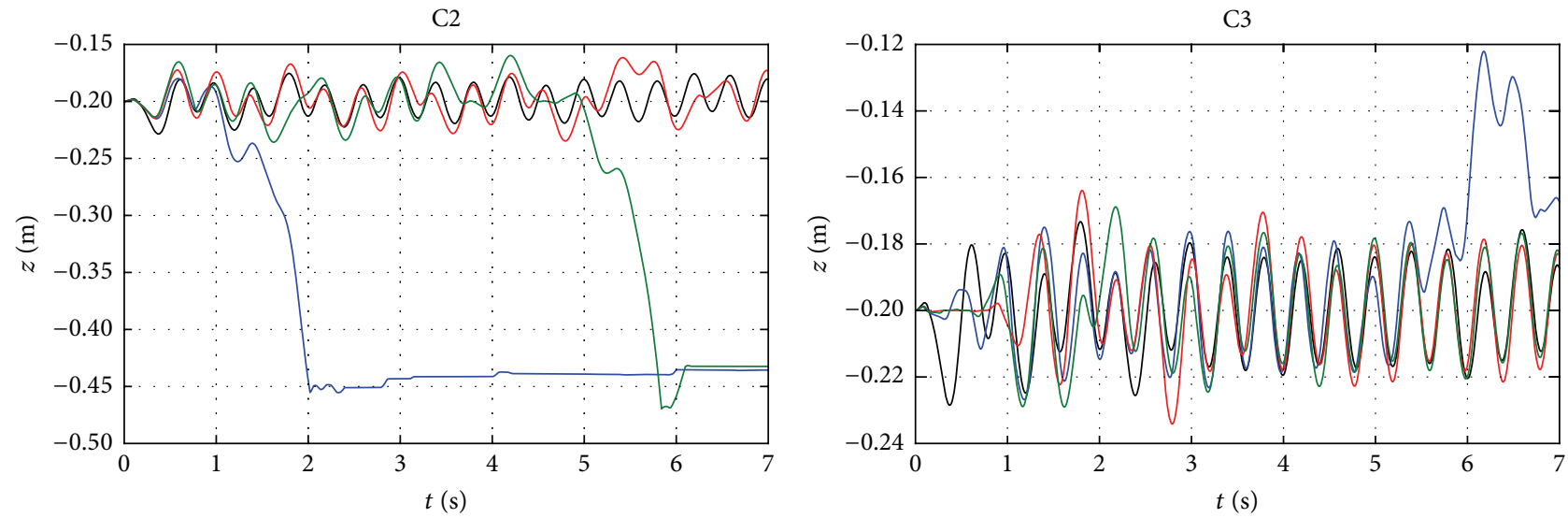

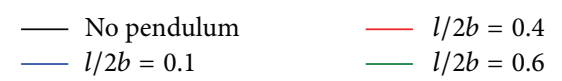

(c)

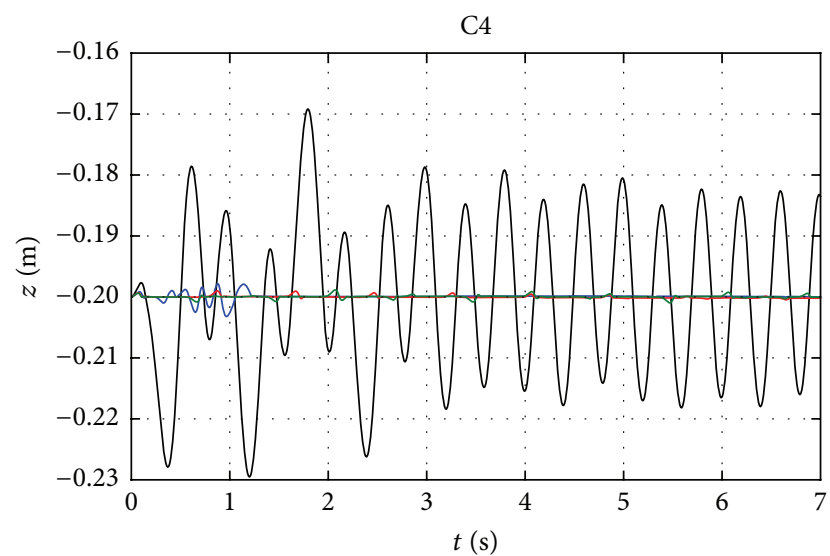

$\begin{array}{ll}- \text { No pendulum } & -l / 2 b=0.4 \\ l / 2 b=0.1 & -l / 2 b=0.6\end{array}$

(e)

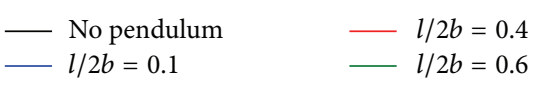

(d)

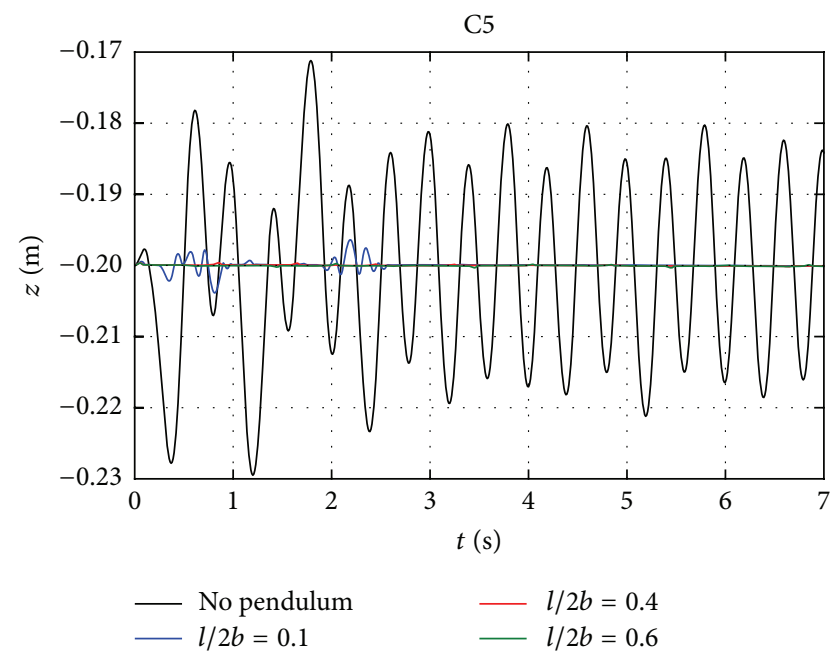

(f)

FIgURE 10: Multibody model and base-block relative motion amplitude $(z)$ under forced oscillations for cases C1, ., C5 of Table 1. 
TABLE 1: Cases studied in the multibody simulations.

\begin{tabular}{lcc}
\hline Case & Mass ratio $\mu=m / M(\%)$ & Length ratio $\lambda=l / 2 b$ \\
\hline C1 & 5 & $0.1-0.4-0.6$ \\
C2 & 10 & $0.1-0.4-0.6$ \\
C3 & 20 & $0.1-0.4-0.6$ \\
C4 & 30 & $0.1-0.4-0.6$ \\
C5 & 40 & $0.1-0.4-0.6$ \\
\hline
\end{tabular}

and to the fixed floor. A collision detection algorithm finds the contact points at each time step and feeds them into the CCP solver, for advancing the DVI integration. A friction model of Amontons-Coulomb type is associated with each contact point, thus automatically taking into account the stick-slip effects. In the presented simulations, we used static and dynamic friction coefficients $\mu_{s}=\mu_{d}=0.6$.

The density of the simulated blocks is $2028 \mathrm{~kg} / \mathrm{m}^{3}$, and the motion of the floor is defined via a rheonomic constraint that imposes a harmonic horizontal motion along the horizontal $z$-axis.

In this case, we used a cosine wave with frequency $f_{z}=$ $2.5 \mathrm{~Hz}$ and amplitude $A_{z}=0.015 \mathrm{~m}$. Various ratios of pendulum lengths, masses, and horizontal frequency have been simulated, obtaining results that, although not exhibiting a perfectly steady-state periodic pattern in all cases because of the numerical nature of the simulation, can confirm the prediction of the analytical model regarding the beneficial effect of the pendulum. This accordance emphasizes the importance of preliminary analysis of real situations using the results of our analytical approach.

Table 1 summarizes this set of cases, and Figure 10 illustrates the main results, that is, the block-table relative motion during the simulation. It is shown that the two heaviest pendulums (mass over $20 \%$ of the block mass) are able to prevent the rocking of the block, whereas in the absence of the pendulum the block would oscillate noticeably. It is a matter of fact that heavy pendulums dramatically reduce oscillations; we point out that these simulations, done taking into account damping and slender structures, are quite new in the rocking-block literature.

Moreover, the simulations show that for a low mass pendulum (i.e., up to $20 \%$ of the block mass) no oscillations reduction occurs despite the pendulum length. It is worth noticing that the effect of length of the pendulum is nearly negligible, with the pendulum mass being the most important parameter for this frequency of "table" oscillation. Note that, due to friction sensitivity of the system, case $\mathrm{C} 2$ presents the overturning of the block.

We finally remark that the numerical method is able to simulate transient phenomena that are not considered in the analytical model and that, optionally, accelerograms can be assigned to all three directions of the floor, thereby simulating a real earthquake.

\section{Conclusions}

The rocking-block problem has been investigated under different points of view, as discussed in Section 1.
Before plotting a synthesis of the obtained results, it is important to state that this is a first step into a field that is not yet well explored. In fact, while several works deal with the "rocking-block" problem, none of them explores the possibility of adding a pendulum to the rocking block with the aim of controlling the oscillations.

We followed two main steps. First and of great interest in this work are the forced oscillations of a block with a pendulum but without friction. Second, as a first step towards general damping, we studied free and forced oscillations of a block without a pendulum but in the presence of dry friction.

Analyzing the problem of the forced oscillations of a rocking block connected with a pendulum, Bubnov-Galerkin's method was applied and the analytical results are exposed in terms of gain-frequency and phase-frequency characteristics. It is of great interest to notice that the presence of the pendulum greatly reduces the amplitude of vibrations in those cases, when the frequency of excitation is not within the resonance area of pendulum. This means that it would be possible to study some passive tuned pendulum to be added to real "rocking blocks" like ancient towers, and so forth, in order to reduce oscillations induced by wind, earthquakes, and so on.

Adding damping, we investigated two situations, both with no pendulum. At first, the case of the free oscillations of a block with dry friction is analytically solved. This solution has been carried out considering small tilt angles. The main results, amply reported in this work, are presented in graphs where the amplitude of the free oscillations is plotted versus frequency. It has to be remarked that, for blocks presenting low base/highness ratios, the theoretical and numerical results are in good accordance. When forcing is added, given by the movement of the base, we still apply Bubnov-Galerkin's method. Starting from certain amplitude, we can define a pseudo resonant frequency and we investigate the block motion in terms of gain-frequency and phasefrequency characteristics, as reported in Figures 8 and 9, which give a good scenario for the influence of an added pendulum on the frequency response of the system. Also in this case, there is good accordance between the theoretical and numerical results.

Finally, a multibody dynamical simulation has been compared against theoretical numerical results on a real case, giving a very satisfactory reduction of oscillations.

\section{Conflict of Interests}

The authors declare that there is no conflict of interests regarding the publication of this paper.

\section{References}

[1] R. W. Clough and J. Penzien, Dynamics of Structures, McGrawHill, New York, NY, USA, 1993.

[2] G. W. Housner, "The behavior of inverted pendulum structures during earthquakes," Bulletin of the Seismological Society of America, vol. 53, no. 2, pp. 403-417, 1963.

[3] M. J. N. Priestley, R. J. Evison, and A. J. Carr, "Seismic response of structures free to rock on their foundation," Bulletin of the 
New Zealand Society for Earthquake Engineering, vol. 11, no. 3, pp. 141-150, 1978.

[4] M. Aslam, W. G. Fodden, and D. T. Scalise, "Earthquake rocking response of rigid bodies," Journal of the Structural Division, vol. 106, no. 2, pp. 377-392, 1980.

[5] C.-S. Yim, A. K. Chopra, and J. Penzien, "Rocking response of rigid blocks to earthquakes," Earthquake Engineering \& Structural Dynamics, vol. 8, no. 6, pp. 565-587, 1980.

[6] Y. Ishiyama, Review and Discussion on Overturning of Bodies by Earthquake Motion, Building Research Institute, Ministry of Construction, 1980.

[7] P. D. Spanos and A.-S. Koh, "Rocking of rigid blocks due to harmonic shaking," Journal of Engineering Mechanics, vol. 110, no. 11, pp. 1627-1642, 1984.

[8] P. R. Lipscombe and S. Pellegrino, "Free rocking of prismatic blocks," Journal of Engineering Mechanics, vol. 119, no. 7, pp. 1387-1410, 1993.

[9] Y. Ishiyama, "Motions of rigid bodies and criteria for overturning by earthquake excitations," Earthquake Engineering \& Structural Dynamics, vol. 10, no. 5, pp. 635-650, 1982.

[10] M. Kaneko and Y. Hayashi, "A proposal for simple equations to express a relation between overturning ratios of rigid bodies and input excitations," in Proceedings of the 13th World Conference on Earthquake Engineering, Vancouver, Canada, August 2004.

[11] R. L. Boroschek and D. Romo, "Overturning criteria for nonanchored non-symmetric rigid bodies," in Proceedings of the 13th World Conference on Earthquake Engineering, Vancouver, Canada, August 2004.

[12] S. J. Hogan, "Rigid block dynamics confined between sidewalls," Philosophical Transactions of the Royal Society A: Physical Sciences and Engineering, vol. 347, no. 1683, pp. 411-419, 1994.

[13] S. J. Hogan, "Slender rigid block motion," Journal of Engineering Mechanics, vol. 120, no. 1, pp. 11-24, 1994.

[14] S. J. Hogan, "On the dynamics of rigid-block motion under harmonic forcing," Proceedings of the Royal Society of London Series A: Mathematical, Physical and Engineering Sciences, vol. 425, no. 1869, pp. 441-476, 1989.

[15] S. J. Hogan, "The many steady state responses of a rigid block under harmonic forcing," Earthquake Engineering \& Structural Dynamics, vol. 19, no. 7, pp. 1057-1071, 1990.

[16] C. M. Wong and W. K. Tso, "Steady state rocking response of rigid blocks. Part 2. Experiment," Earthquake Engineering \& Structural Dynamics, vol. 18, no. 1, pp. 107-120, 1989.

[17] C. Yilmaz, M. Gharib, and Y. Hurmuzlu, "Solving frictionless rocking block problem with multiple impacts," Proceedings of the Royal Society of London Series A: Mathematical, Physical and Engineering Sciences, vol. 465, no. 2111, pp. 3323-3339, 2009.

[18] E. G. Dimitrakopoulos, M. J. DeJong, and E. G. Dimitrakopoulos, "Revisiting the rocking block: closed-form solutions and similarity laws," Proceedings of the Royal Society of London A, vol. 468, no. 2144, pp. 2294-2318, 2012.

[19] G. W. Housner, L. A. Bergman, T. K. Caughey et al., "Structural control: past, present, and future," Journal of Engineering Mechanics, vol. 123, no. 9, pp. 897-971, 1997.

[20] S. Lenci and G. Rega, "Optimal control and anti-control of the nonlinear dynamics of a rigid block," Philosophical Transaction of the Royal Society A, vol. 364, no. 1846, pp. 2353-2381, 2006.

[21] D. E. Stewart and J.-S. Pang, "Differential variational inequalities," Mathematical Programming, vol. 113, no. 2, pp. 345-424, 2008.
[22] M. Anitescu and A. Tasora, "An iterative approach for cone complementarity problems for nonsmooth dynamics," Computational Optimization and Applications, vol. 47, no. 2, pp. 207235, 2010.

[23] A. Tasora and M. Anitescu, "A matrix-free cone complementarity approach for solving large-scale, nonsmooth, rigid body dynamics," Computer Methods in Applied Mechanics and Engineering, vol. 200, no. 5-8, pp. 439-453, 2011.

[24] D. Negrut, A. Tasora, H. Mazhar, T. Heyn, and P. Hahn, "Leveraging parallel computing in multibody dynamics," Multibody System Dynamics, vol. 27, no. 1, pp. 95-117, 2012. 


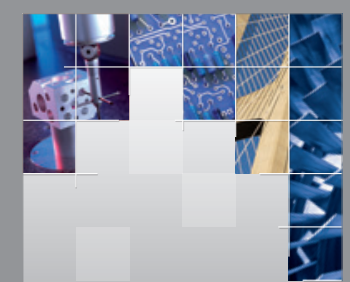

\section{Enfincering}
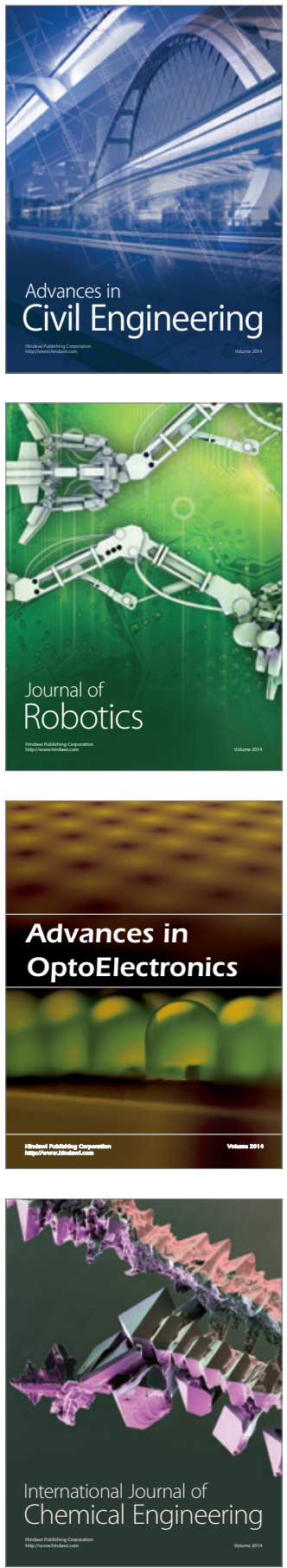

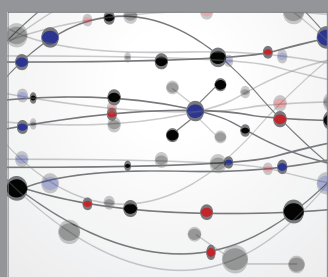

The Scientific World Journal

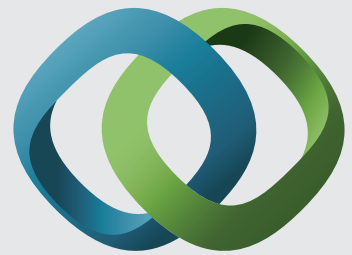

\section{Hindawi}

Submit your manuscripts at

http://www.hindawi.com
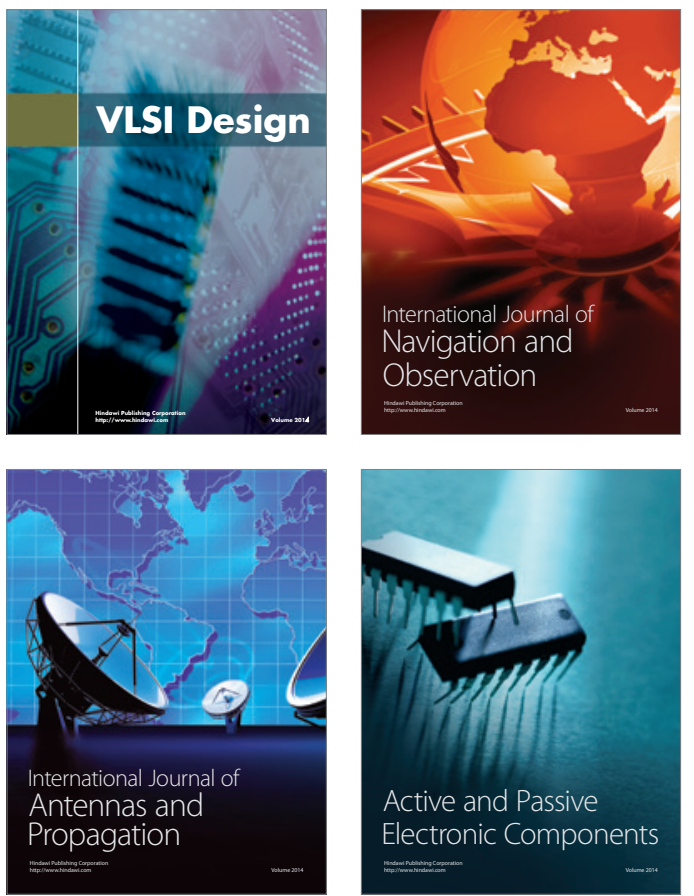
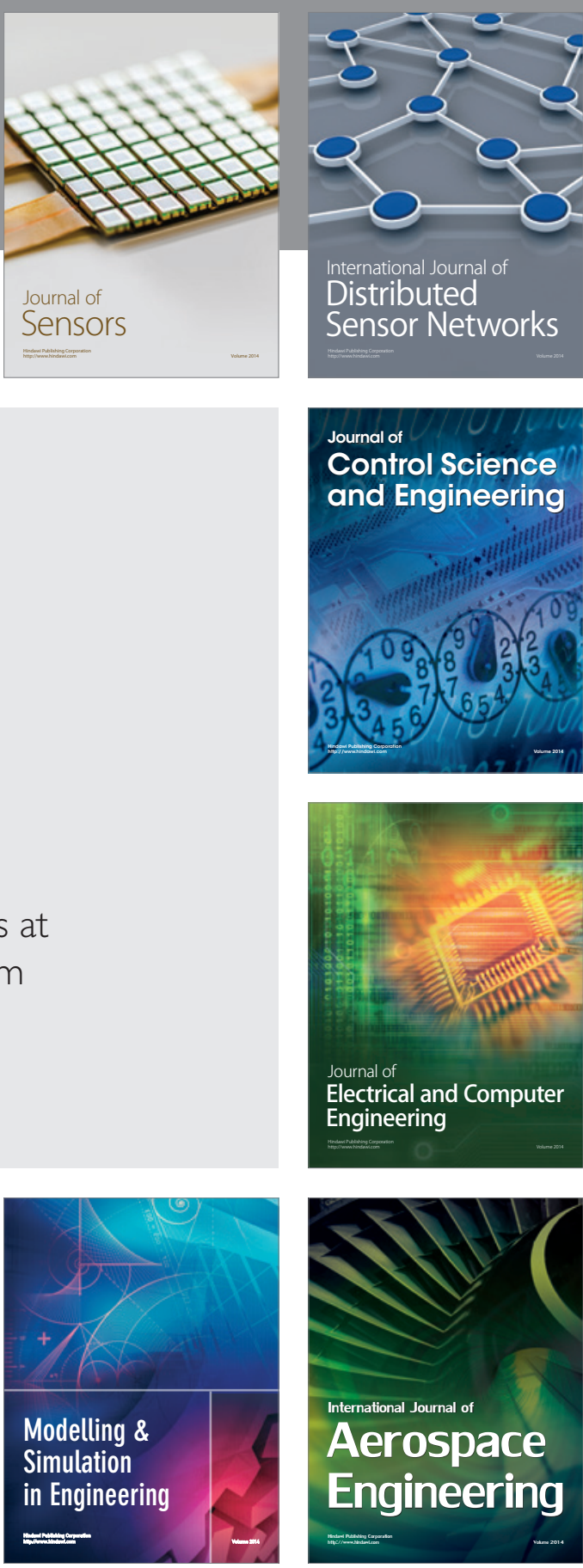

International Journal of

Distributed

Sensor Networks

Journal of

Control Science

and Engineering
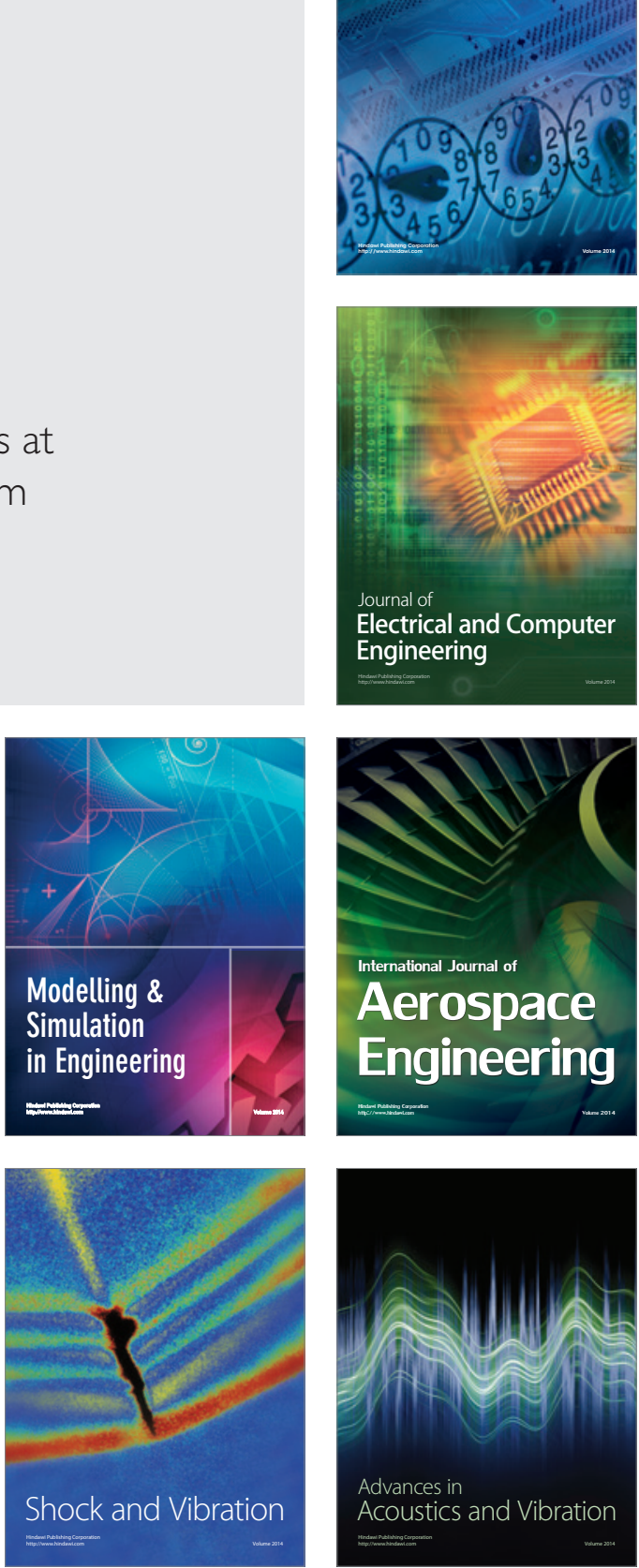